\title{
Language Recognition via Sparse Coding
}

\section{Citation}

Gwon, Youngjune L., William M. Campbell, Douglas E. Sturim, and H. T. Kung. 2016. "Language Recognition via Sparse Coding." In Proc. Interspeech 2016, pp. 2920-2924.

\section{Published Version}

doi:10.21437/Interspeech.2016-881

\section{Permanent link}

http://nrs.harvard.edu/urn-3:HUL.InstRepos:33973840

\section{Terms of Use}

This article was downloaded from Harvard University's DASH repository, and is made available under the terms and conditions applicable to Open Access Policy Articles, as set forth at http:// nrs.harvard.edu/urn-3:HUL.InstRepos:dash.current.terms-of-use\#OAP

\section{Share Your Story}

The Harvard community has made this article openly available.

Please share how this access benefits you. Submit a story.

Accessibility 


\title{
Language Recognition via Sparse Coding ${ }^{\dagger}$
}

\author{
Youngjune L. Gwon ${ }^{1}$, William M. Campbell ${ }^{1}$, Douglas Sturim ${ }^{1}$, H. T. Kung ${ }^{2}$ \\ ${ }^{1}$ MIT Lincoln Laboratory \\ ${ }^{2}$ Harvard University \\ gyjell.mit.edu, wcampbell@ll.mit.edu, sturimell.mit.edu, kung@harvard.edu
}

\begin{abstract}
Spoken language recognition requires a series of signal processing steps and learning algorithms to model distinguishing characteristics of different languages. In this paper, we present a sparse discriminative feature learning framework for language recognition. We use sparse coding, an unsupervised method, to compute efficient representations for spectral features from a speech utterance while learning basis vectors for language models. Differentiated from existing approaches in sparse representation classification, we introduce a maximum a posteriori (MAP) adaptation scheme based on online learning that further optimizes the discriminative quality of sparse-coded speech features. We empirically validate the effectiveness of our approach using the NIST LRE 2015 dataset.
\end{abstract}

Index Terms: speech recognition, sparse coding

\section{Introduction}

Originally used to explain neuronal activations [1], sparse coding emerges as an effective means to discover underlying structures of unknown data. High-level feature representations learned from sparse coding occasionally have resulted the best performance for discriminative tasks in computer vision. Yet, sparse coding of speech features - or audio signals in generalhas not been explored to its full potential. In this paper, we investigate a discriminative learning framework based on sparse coding for language recognition.

Language recognition refers to a systematic process of identifying the spoken language in a speech utterance. Over the years, Gaussian mixture models (GMMs) [2] and support vector machine (SVM) [3] have been crucial to build a highperformance language identification (LID) system. More recently, the idea of total variability space or i-vector [4] has been studied for LID. Motivated by joint factor analysis (JFA) for speaker verification [5], i-vector approaches are known to produce state-of-the-art results in language recognition. A conventional GMM-based language model uses the shifted delta cepstra (SDC) feature computed from a linear expansion of consecutive MFCC blocks. A more contemporary approach for acoustic feature extraction is to train a deep neural network (DNN) for bottleneck feature (BNF) $[6,7,8,9]$. Currently, the best LID systems are built on i-vector modeling of the DNN BNFs.

Sparse coding has been previously applied to speaker and language identification $[10,11,12]$. Despite much interest from the machine learning community, there is surprisingly little work in sparse coding for speech. A sparse coding-based classification pipeline can take a simple classifier such as linear SVM

\footnotetext{
${ }^{\dagger}$ This work was sponsored by the Department of Defense under Air Force Contract FA8721-05-C-0002. Opinions, interpretations, conclusions, and recommendations are those of the authors and are not necessarily endorsed by the United States Government.
}

trained on the sparse feature vectors. However, it is known to perform on par with (or better than) more complex nonlinear schemes (e.g., deep neural networks, kernel SVM) [13]. One possible explanation is that sparse coding can achieve a nearoptimal approximation of much complicated nonlinear relationship through local and piecewise linear functions.

We structure the rest of this paper as follows. In Section 2, we present a background on sparse coding. Section 3 describes our sparse coding-based approaches for language recognition. In particular, we propose adaptive sparse coding (ASC), an enhancement to the semi-supervised classification pipeline on vanilla sparse coding, and discuss an online method for perutterance dictionary adaptation. As a result, we can significantly improve the discriminative quality of sparse-coded speech features. In Section 4, we evaluate the proposed approaches against i-vector based benchmark pipelines developed by Lincoln Laboratory and MIT on a subset of the NIST LRE 2015 comprising the Arabic and Chinese clusters. Section 5 concludes the paper.

\section{Sparse Coding Background}

Sparse coding is an unsupervised method to learn an efficient representation of data using a small number of basis vectors. It has been used to discover higher-level features present in data from unlabeled examples. Given an example $\mathbf{x} \in \mathbb{R}^{N}$, sparse coding searches for a representation $\mathbf{y} \in \mathbb{R}^{K}$ (i.e., the feature vector for $\mathbf{x}$ ) while simultaneously updating the dictionary $\mathbf{D} \in$ $\mathbb{R}^{N \times K}$ of $K$ basis vectors by

$$
\min _{\mathbf{D}, \mathbf{y}}\|\mathbf{x}-\mathbf{D y}\|_{2}^{2}+\lambda\|\mathbf{y}\|_{1} \quad \text { s.t. }\left\|\mathbf{d}_{i}\right\|_{2} \leq 1, \forall i
$$

where $\mathbf{d}_{i}$ is $i$ th dictionary atom in $\mathbf{D}$, and $\lambda$ is a regularization parameter that penalizes over the $\ell_{1}$-norm, which induces a sparse solution. With $K>N$, sparse coding typically trains an overcomplete dictionary. This makes the sparse code $\mathbf{y}$ higher in dimension than $\mathbf{x}$, but only $S \ll N$ elements in $\mathbf{y}$ are nonzero.

A more direct way to control sparsity is to regularize on the $\ell_{0}$ pseudo-norm $\|\mathbf{y}\|_{0}$, describing the number of nonzero elements in $\mathbf{y}$. However, it is known to be intractable to compute the sparsest $\ell_{0}$ solution in general. The approach in Eq. (1) is called least absolute shrinkage and selection operator (LASSO) [14], a convex relaxation of the $\ell_{0}$ sparse coding that induces sparse y's. We use least angle regression (LARS) [15] to solve the LASSO problem. We also consider orthogonal matching pursuit (OMP) [16], a greedy- $\ell_{0}$ sparse coding algorithm that computes an at-most $S$-sparse y extremely fast by

$$
\min _{\mathbf{D}, \mathbf{y}}\|\mathbf{x}-\mathbf{D y}\|_{2}^{2} \quad \text { s.t. }\|\mathbf{y}\|_{0} \leq S \text {. }
$$




\section{Our Approach}

\subsection{Shifted delta cepstral feature extraction}

We use a spectral-based technique by Torres et al. $[17,18]$ to process speech waveforms. Speech is analyzed with a Hamming window of $20-\mathrm{msec}$ duration at a $10-\mathrm{msec}$ frame rate. The windowed speech waveforms pass through a mel-scale filterbank and RASTA filtering with per-utterance normalization to zero mean and unit variance. Using the 7-1-3-7 scheme, we calculate the shifted delta cepstral (SDC) coefficients. Concatenating with static cepstra, the spectral features extracted from speech form a 56-dimensional vector. Lastly, we run energybased speech activity detection to remove undesirable background noise.

\subsection{Vanilla sparse coding}

The key reasoning for sparse coding is to learn useful representations by decomposing spectro-temporal features of speech into a sparse linear combination of basis vectors in a dictionary (also learned). Nonzeros in the computed sparse code quantify the presence of specific basis vectors. By exploiting variation of the nonzero locations and magnitude, we can build a discriminative pipeline for language recognition.

Figure 1 describes a baseline sparse coding approach for LID, which we call "vanilla sparse coding (VSC)." VSC is a semi-supervised approach. Assuming $L$ languages of interest $\mathcal{L} \in\left\{l_{1}, \ldots, l_{L}\right\}$, we perform sparse coding with an unbiased mix of unlabeled speech examples from all languages to train a dictionary $\mathbf{D} \in \mathbb{R}^{N \times K}$ during the unsupervised phase. The trained dictionary represents universal sparse modeling of the $L$ languages. That is, given an unknown speech input $\mathbf{x} \in \mathbb{R}^{N}$, we can compute its sparse representation $\mathbf{y} \in \mathbb{R}^{K}$ using $\mathbf{D}$. By sparse modeling assumption, $\mathbf{y}$ has only several nonzero elements

$$
\mathbf{x} \approx y_{1} \mathbf{d}_{1}+y_{2} \mathbf{d}_{2}+\cdots+y_{K} \mathbf{d}_{K},
$$

where $y_{j}$ is $j$ th element in $\mathbf{y}, \mathbf{d}_{j}$ the $j$ th basis vector in $\mathbf{D}$. We use the notation $\mathbf{X}=\left[\mathbf{x}^{(1)} \ldots \mathbf{x}^{(n)}\right]$ for a batch of $n$ unlabeled training examples, where $\mathbf{x}^{(i)} \in \mathbb{R}^{N}$ is the $i$ th example in the batch. Optionally, $\mathbf{X}$ can be normalized and whitened before sparse coding for better result.

The supervised phase uses a labeled dataset. Consider $m$ labeled training examples in $\mathbf{X}_{\ell}=\left[\chi^{(1)} \ldots \chi^{(m)}\right]$. Now, each example $\chi^{(k)}=\left\{\mathbf{x}^{(k)}, l^{(k)}\right\}$ includes a language label $l^{(k)} \in \mathcal{L}$ for $\mathbf{x}^{(k)}$. Recall each $\mathbf{x}$ contains the spectral feature for a single frame (i.e., $10 \mathrm{msec}$ ). Since a speech utterance is much longer (up to minutes), sparse coding will result in too many feature vectors per utterance. Before the supervised training of classifiers, we perform pooling, a technique popularized in computer vision, across all sparse codes from the same utterance. The purpose of pooling is two-fold: 1) aggregation of feature vectors and 2) statistical robustness.

\subsection{Enhancement: adaptive sparse coding}

We propose an enhancement of VSC as illustrated in Figure 2. We name the approach "adaptive sparse coding (ASC)." The unsupervised phase of ASC is identical to VSC, and the dictionary $\mathbf{D}$ for universal sparse modeling of all languages is first learned. The basic idea of ASC is to adapt $\mathbf{D}$ to the utterancedependent dictionary $\mathbf{D}_{\mathrm{a}}$ during the supervised phase. With both $\mathbf{D}$ and $\mathbf{D}_{a}$, we can compute two sparse codes $\mathbf{y}$ and $\mathbf{y}_{\mathbf{a}}$ for each input vector $\mathbf{x}$ from the same utterance such that

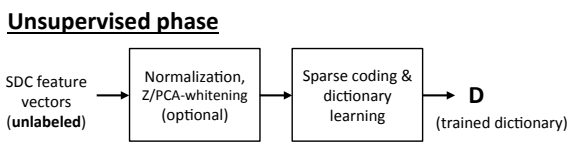

\section{Supervised phase}

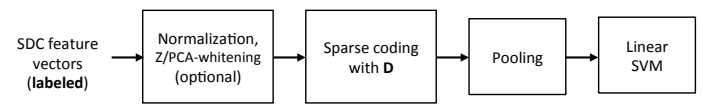

Figure 1: Vanilla sparse coding pipeline

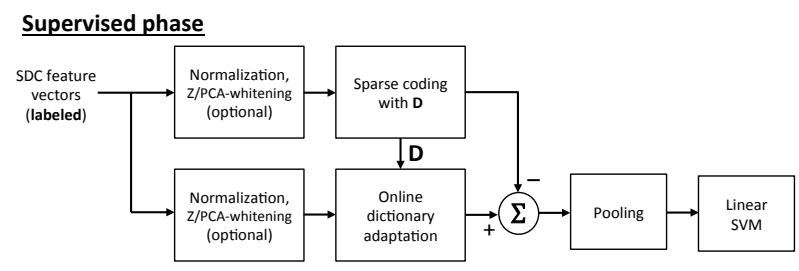

Figure 2: During the supervised phase of adaptive sparse coding pipeline, per-utterance dictionary adaptation is performed. Pooled difference vector between adapted and universal sparse models is used to train classifiers.

$\mathbf{x}=\mathbf{D} \mathbf{y}$ and $\mathbf{x}=\mathbf{D}_{\mathrm{a}} \mathbf{y}_{\mathrm{a}}$, respectively. ASC takes in the difference $\boldsymbol{\Delta}=\mathbf{y}_{\mathrm{a}}-\mathbf{y}$ to train classifiers (compared to $\mathbf{y}$ for VSC as in Figure 1). Note that $\Delta$ vectors from the same utterance are also pooled before applied to classifiers.

Our idea of adapted sparse coding dictionaries and forming discriminative $\Delta$ is analogous to adapted GMM-UBM and supervectors $[2,19]$. Consider a probabilistic model for sparse coding under a Gaussian noise

$$
p(\mathbf{x} \mid \mathbf{D}, \mathbf{y}) \sim \mathcal{N}\left(\sum_{j=1}^{K} y_{j} \mathbf{d}_{j}, \sigma^{2} \mathbf{I}\right)
$$

where the Gaussian noise has a zero-mean and covariance $\sigma^{2} \mathbf{I}$. A sparse prior $p(\mathbf{y}) \propto \prod_{j} e^{-\lambda\left|y_{j}\right|}$ regularizes the activations on sparse code $\mathbf{y}$. Note that the hyperparameter $\lambda$ is same as the regularization parameter of Equation (1). We can formulate the maximum a posteriori (MAP) estimation problem to solve for $\left\{\mathbf{y}_{\mathrm{a}}, \mathbf{D}_{\mathrm{a}}\right\}$ jointly

$$
\arg \max _{\mathbf{D}^{\prime}, \mathbf{y}^{\prime}} p\left(\mathbf{y}^{\prime} \mid \mathbf{x}, \mathbf{D}^{\prime}\right)=\arg \max _{\mathbf{D}^{\prime}, \mathbf{y}^{\prime}} p\left(\mathbf{x} \mid \mathbf{D}^{\prime}, \mathbf{y}^{\prime}\right) p\left(\mathbf{y}^{\prime}\right) .
$$

Since $p\left(\mathbf{x} \mid \mathbf{D}^{\prime}, \mathbf{y}^{\prime}\right)$ is a multivariate Gaussian density function, we can derive an analytical solution for Equation (4). For this paper, however, we focus on efficient estimation of the adapted dictionary and sparse code by following an online method by Mairal et al. [20].

In Algorithm 1, we present a fast online algorithm for dictionary adaptation. This algorithm is guaranteed to converge and computes a good estimate of $\mathbf{D}_{\mathbf{a}}$ from $\mathbf{D}$ given an arbitrary amount of utterance input. In particular, block-coordinate descent in the inner-loop sequentially updates each basis vector (column) in the dictionary. Since the $\mathbf{y}$ vectors are sparse, the coefficients of the matrix $\mathbf{A}$ are concentrated on the diagonal, making the search for optimal $\mathbf{D}_{\mathrm{a}}$ very efficient. For the sparse coding step in the inner-loop, we can use either LARS or OMP. 

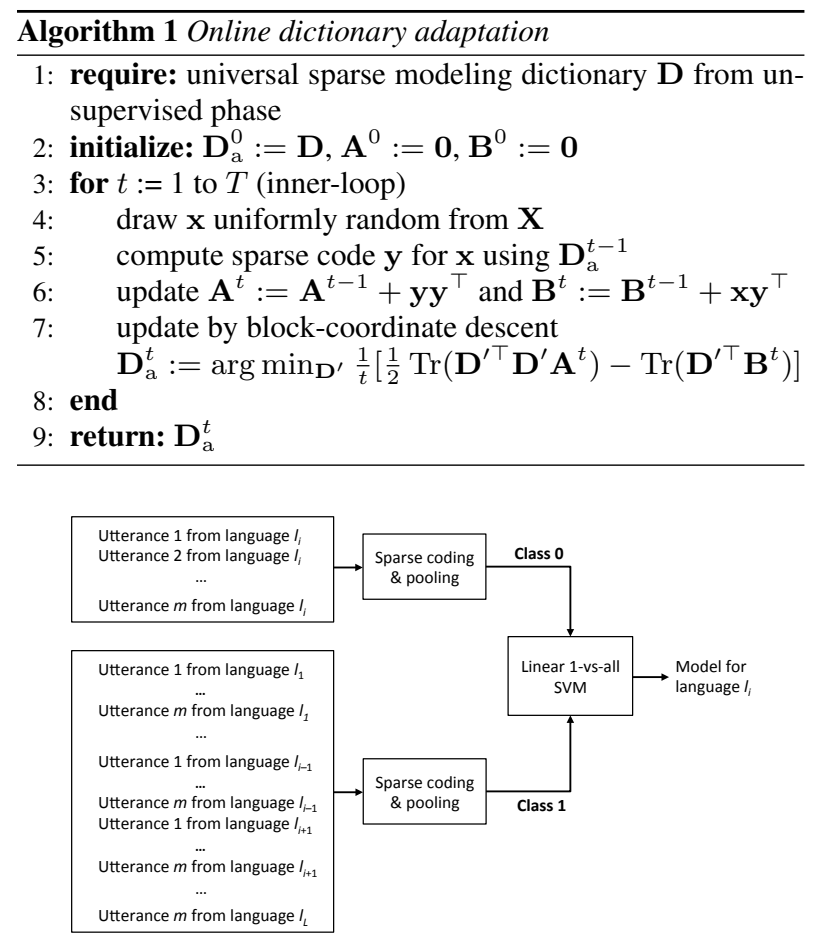

Figure 3: Training 1-vs-all SVM for each language

\subsection{SVM classification}

We consider support vector machines (SVMs) for both VSC and ASC pipelines. The kernel trick for SVM has been studied widely to cope with cases where the input vectors for SVM are not linearly separable. For our case, sparse coding and pooling together give reasonably sufficient nonlinear transformation for the SDC coefficients. Hence, we use off-the-shelf linear SVMs only.

A well-accepted strategy for a LID system is to train $1-v s$ all classifiers as explained in Figure 3. To train the model for language $l_{i} \in \mathcal{L}$, we input the pooled sparse codes for all labeled examples from $l_{i}$ as class 0 . For utterances from all other languages $l_{j} \in \mathcal{L}_{\backslash l_{i}}$, we use class 1 .

\section{Experiments}

\subsection{Task, dataset, and evaluation metrics}

To evaluate the performance of sparse coding pipelines for LID, we consider the NIST Language Recognition Evaluation (LRE) 2015 [21]. The task is to determine the average performance of a LID system that can classify each language as a target within six predefined language clusters. The language clusters are Arabic, Chinese, English, French, Slavic, and Iberian with 20 different languages in total. For the time being, we present a partial evaluation focusing only on the Arabic and Chinese clusters. As summarized in Table 1, there are 5 languages from Arabic and 4 languages from Chinese in NIST LRE 2015.

The NIST dataset comes in training and evaluation subsets. We cut the training subset into train and dev. Using train, we do sparse coding and dictionary learning. SVM supervised training for each language is also done with train. The size of dev is approximately $\frac{1}{4}$ of train, and we reserve dev for calibration and fusion at the backend, which will be discussed in
Table 1: Arabic and Chinese language clusters from NIST LRE 2015

\begin{tabular}{|l|r|}
\hline Cluster & Target languages \\
\hline \hline Arabic & Egyptian, Iraqi, Levantine, Maghrebi, Modern Standard \\
\hline Chinese & Cantonese, Mandarin, Min, Wu \\
\hline
\end{tabular}

$\S 4.3$. The amount of training examples for each language is uneven. It ranges from 2.6 (zho-yue) to 97.5 hours (ara-arz) in speech duration.

The eval subset serves as held-out test data to evaluate the language recognition performance (i.e., classification cost). Following the 2015 evaluation plan [22], we adopt the NIST average cost performance as our evaluation metric

$$
\begin{aligned}
C_{\mathrm{avg}} & =\frac{1}{N_{L}}\left\{\left[C_{\mathrm{miss}} \cdot p_{\text {target }} \cdot \sum_{l_{T}} p_{\mathrm{miss}}\left(l_{T}\right)\right]\right. \\
& \left.+\frac{1}{N_{L}-1}\left[C_{\mathrm{FA}} \cdot\left(1-p_{\text {target }}\right) \cdot \sum_{l_{T}} \sum_{l_{N}} p_{\mathrm{FA}}\left(l_{T}, l_{N}\right)\right]\right\} .
\end{aligned}
$$

\subsection{Methods and training}

For comparative performance evaluation, we have trained two benchmark pipelines on an i-vector framework [4], IVEC-SDC and IVEC-BNF. For years, i-vector based systems have been able to produce the top results in numerous speaker and language recognition challenges. IVEC-SDC takes in the same 71-3-7 SDC feature vectors used for our VSC and ASC pipelines. IVEC-BNF, however, uses a 64-dimensional bottleneck feature (BNF) obtained by training a deep neural network (DNN). The DNN has 7 hidden layers, all of which have 1,024 neurons except the sixth layer that has 64 neurons with a linear activation function firing the BNFs. We remark that both our benchmark pipelines IVEC-SDC and IVEC-BNF are the part of MIT Lincoln Laboratory's NIST LRE 2015 submission [23].

Before sparse coding, we normalize each input vector by removing its mean and dividing by the standard deviation. The normalized input vectors are then ZCA-whitened [24] without a dimensionality reduction. Our choice of using ZCA-whitening over PCA-whitening has been determined empirically.

We have tested multiple configurations of VSC and ASC by varying the choice of sparse coding algorithm, $\ell_{1}$-regularized LARS or greedy- $\ell_{0}$ OMP, and the number of basis vectors in a dictionary $K=512,1024$. Since we have applied only the SDC features to sparse coding, we add a "-SDC" prefix to name our methods. For example, ASC-LARS-1024-SDC denotes adaptive sparse coding with LARS and a 1,024-basis vector dictionary on the SDC features. Throughout our experiments with LARS, we use a sparsity penalty $\lambda=0.15$. For OMP, we use a sparsity bound $S=0.1 \times 56 \approx 6$. Our implementation is done in MATLAB, using the INRIA SPAMS (SPArse Modeling Software) [25] for LARS and the Technion toolbox [26] for OMP. We use LIBSVM [27].

During the unsupervised phase, we learn $\mathbf{D}$ for universal sparse modeling of all 9 languages. During the supervised phase, we perform a five-fold cross-validation on train to determine hyperparameters of the SVMs. For ASC, we also adapt $\mathbf{D}$ to utterance-specific dictionaries using the train subset during the supervised phase. We have decided to go with average pooling after trying out both average and max pooling methods described below.

$$
\text { 1. Average: } f\left(\left\{\mathbf{y}^{(1)}, \ldots, \mathbf{y}^{(M)}\right\}\right)=\frac{1}{M} \sum_{j=1}^{M}\left|\mathbf{y}^{(j)}\right|
$$


2. $\begin{aligned} \operatorname{Max}: & f\left(\left\{\mathbf{y}^{(1)}, \ldots, \mathbf{y}^{(M)}\right\}\right) \\ & =\max _{\forall k}\left(\left\{\left|y_{k}^{(1)}\right|, \ldots,\left|y_{k}^{(M)}\right|\right\}\right)\end{aligned}$

\subsection{Fusion}

The historical NIST LREs have found that running multiple pipelines concurrently can be beneficial. Thus, we consider post-processing at the backend that consists of per-pipeline calibration and fusion using the dev subset. We perform a simple linear fusion with one of the sparse coding pipelines and IVECSDC

$$
l l r_{\text {fusion }}^{l_{T}}=\rho \cdot \frac{l l r_{1}^{l_{T}}-\mu_{1}^{l_{T}}}{\sigma_{1}^{l_{T}}}+(1-\rho) \cdot \frac{l l r_{2}^{l_{T}}-\mu_{2}^{l_{T}}}{\sigma_{2}^{l_{T}}} .
$$

Here, we use a mixing ratio $\rho$ to combine the scores (i.e., loglikelihood ratios $l l r_{1}$ and $l l r_{2}$ with respect to a target language $l_{T}$ ) from the two pipelines. More sophisticated fusion schemes such as logistic regression and neural networks can also be used.

\subsection{Results and discussion}

Table 2 presents the performance comparison on the average cost metric $C_{\text {avg }}$ for the i-vector benchmarks, as well as the proposed VSC and ASC pipelines. These results are obtained by running the eval subset, which includes 34,530 utterances for Arabic and 44,596 utterances for Chinese. The bold-faced numbers represent the best result from each IVEC, VSC, ASC group.

Using the exact same SDC features, our best sparse coding pipeline ASC-LARS-1024-SDC significantly outperforms the IVEC-SDC approach for both language clusters. This result shows that our proposed sparse-modeling approach has significant potential. With different features, the IVEC-BNF, is able to outperform our proposed method. Our method still remains competitive on the Arabic cluster with a $C_{\text {avg }}$ of 0.1874 on the Arabic cluster compared to 0.1811 for IVEC-BNF. This result points to future work where we apply the sparse technique to BN features.

We find that ASC makes a significant improvement over VSC. If the choice of sparse coding algorithm and the number of basis vectors in a dictionary $K$ were the same, ASC results in consistently better cost performance. Overcompleteness of sparse coding dictionary is an important hyperparameter for both VSC and ASC. Increasing $K$ from 512 to 1,024 has always improved the cost performance. Also for both pipelines, LARS results in a better performance. However, the average computation time for LARS is found an order of magnitude higher than OMP.

In Table 3, we report improved cost performance by the linear fusion of our VSC and ASC pipelines with IVEC-SDC. For calibration, we have varied the fusion hyperparameter $\rho$ from 0.1 to 0.9 and chosen the value that results the best cost performance on the dev dataset. We have found 0.5 for VSC-LARS1024-SDC and 0.6 for ASC-LARS-1024-SDC. Fusion enables us to achieve the new best $C_{\text {avg }}$ for both Arabic and Chinese. In particular, $C_{\text {avg }}$ of 0.1652 for Arabic is better than IVEC-BNF.

\section{Conclusion and Future Work}

Sparse coding has achieved great performances for discriminative tasks in computer vision and object recognition. Despite its growing interest, sparse coding has relatively little work known in speech. In this paper, we have described semi-supervised approaches for sparse coding on the task of language recognition.
Table 2: Comparison of average cost performance $\left(C_{\mathrm{avg}}\right)$ on Arabic and Chinese language clusters

\begin{tabular}{lcc}
\hline Pipeline & Arabic & Chinese \\
\hline \hline IVEC-SDC & 0.2566 & 0.2054 \\
IVEC-BNF & $\mathbf{0 . 1 8 1 1}$ & $\mathbf{0 . 1 1 6 0}$ \\
\hline \hline VSC-LARS-512-SDC & 0.2615 & 0.2556 \\
VSC-OMP-512-SDC & 0.2823 & 0.2699 \\
VSC-LARS-1024-SDC & $\mathbf{0 . 2 3 9 3}$ & $\mathbf{0 . 2 0 4 3}$ \\
VSC-OMP-1024-SDC & 0.2486 & 0.2120 \\
\hline ASC-LARS-512-SDC & 0.2187 & 0.1909 \\
ASC-OMP-512-SDC & 0.2342 & 0.2036 \\
ASC-LARS-1024-SDC & $\mathbf{0 . 1 8 7 4}$ & $\mathbf{0 . 1 6 3 4}$ \\
ASC-OMP-1024-SDC & 0.2015 & 0.1983 \\
\hline
\end{tabular}

Table 3: Cost performance improvement by linear fusion

\begin{tabular}{|l|c|c|}
\hline Fusion scheme & Arabic & Chinese \\
\hline \hline VSC-LARS-1024-SDC + IVEC-SDC & 0.1988 & 0.1857 \\
ASC-LARS-1024-SDC + IVEC-SDC & $\mathbf{0 . 1 6 5 2}$ & $\mathbf{0 . 1 2 2 6}$ \\
\hline
\end{tabular}

Differentiated from existing approaches in sparse representation classification, we propose the MAP adaptation on the dictionary for sparse modeling of each language to improve the discriminative quality of sparse-coded speech features. Using the NIST LRE 2015 dataset, we experimentally validate the effectiveness of our approaches. Also, empirical results in the backend fusion indicate that sparse coding, ASC in particular, can be a viable component for a top LID system.

Our immediate future work includes the full evaluation of the NIST LRE dataset. We plan to explore the ASC hyperparameter space in a more elaborated manner. As an initial approach, we have opted to focus on the SDC feature input only. Although a direct comparison to the IVEC-BNF benchmark may not be too meaningful at the moment, the superior performance by VSC and ASC over IVEC-SDC leads us to the next logical step in trying out the DNN bottleneck features.

\section{References}

[1] B. Olshausen and D. Field, "Sparse Coding with an Overcomplete Basis Set: Strategy Employed by V1?" Vision research, 1997.

[2] D. A. Reynolds, T. F. Quatieri, and R. B. Dunn, "Speaker Verification Using Adapted Gaussian Mixture Models," Digital Signal Processing, vol. 10, no. 1-3, pp. 19-41, 2000.

[3] W. M. Campbell, J. P. Campbell, D. A. Reynolds, E. Singer, and P. A. Torres-Carrasquillo, "Support Vector Machines for Speaker and Language Recognition," Computer Speech \& Language, vol. 20, no. 2-3, pp. 210-229, 2006.

[4] N. Dehak, P. A. Torres-Carrasquillo, D. A. Reynolds, and R. Dehak, "Language Recognition via i-Vectors and Dimensionality Reduction," in INTERSPEECH, 2011.

[5] P. Kenny, P. Ouellet, N. Dehak, V. Gupta, and P. Dumouchel, "A Study of Interspeaker Variability in Speaker Verification," IEEE Trans. on Audio, Speech, and Language Processing, vol. 16, no. 5, pp. 980-988, July 2008.

[6] S. Yaman, J. W. Pelecanos, and R. Sarikaya, "Bottleneck Features for Speaker Recognition," in Odyssey, 2012.

[7] Y. Song, B. Jiang, Y. Bao, S. Wei, and L. R. Dai, "i-Vector Representation Based on Bottleneck Features for Language Identification," Electronics Letters, vol. 49, no. 24, pp. 1569-1570, November 2013.

[8] F. Richardson, D. A. Reynolds, and N. Dehak, "A Unified Deep Neural Network for Speaker and Language Recognition," in $I N$ TERSPEECH, 2015. 
[9] L. Ferrer, Y. Lei, M. McLaren, and N. Scheffer, "Study of Senonebased Deep Neural Network Approaches for Spoken Language Recognition," IEEE/ACM Trans. on Audio, Speech and Language Processing, vol. 24, no. 1, pp. 105-116, 2016.

[10] G. Sivaram, S. K. Nemala, M. Elhilali, T. D. Tran, and H. Hermansky, "Sparse Coding for Speech Recognition," in ICASSP, 2010.

[11] T. N. Sainath, B. Ramabhadran, M. Picheny, D. Nahamoo, and D. Kanevsky, "Exemplar-based Sparse Representation Features: From TIMIT to LVCSR," IEEE Trans. on Audio, Speech, and Language Processing, vol. 19, no. 8, pp. 2598-2613, November 2011.

[12] O. Vinyals and L. Deng, "Are Sparse Representations Rich Enough for Acoustic Modeling?” in INTERSPEECH, 2012.

[13] A. Coates, A. Y. Ng, and H. Lee, "An Analysis of Single-layer Networks in Unsupervised Feature Learning," in AISTATS, 2011.

[14] R. Tibshirani, "Regression Shrinkage and Selection via Lasso," Journ. of Royal Statistical Society, 1994.

[15] B. Efron, T. Hastie, L. Johnstone, and R. Tibshirani, "Least Angle Regression," Annals of Statistics, 2004.

[16] J. Tropp and A. Gilbert, "Signal Recovery From Random Measurements via Orthogonal Matching Pursuit," IEEE Trans. on Information Theory, 2007.

[17] P. A. Torres-Carrasquillo, E. Singer, M. A. Kohler, R. J. Greene, D. A. Reynolds, and J. R. Deller Jr, "Approaches to Language Identification Using Gaussian Mixture Models and Shifted Delta Cepstral Features,' in INTERSPEECH, 2002.

[18] D. A. Reynolds, W. M. Campbell, W. Shen, and E. Singer, "Automatic Language Recognition via Spectral and Token Based Approaches," in Springer Handbook of Speech Processing and Communication. Springer-Verlag GmbH, 2007.

[19] W. M. Campbell, D. E. Sturim, D. A. Reynolds, and A. Solomonoff, "SVM Based Speaker Verification Using a GMM Supervector Kernel and NAP Variability Compensation," in ICASSP, 2006.

[20] J. Mairal, F. Bach, J. Ponce, and G. Sapiro, "Online Dictionary Learning for Sparse Coding," in ICML, 2009.

[21] National Institute of Science and Technology (NIST), "2015 Language Recognition Evaluation," http://www.nist.gov/itl/iad/mig/lre15.cfm.

[22] $—$ "The $2015 \quad$ NIST $\quad$ Language Recognition Evaluation Plan (LRE15), http://www.nist.gov/itl/iad/mig/upload/LRE15_EvalPlan_v23.pdf.

[23] P. A. Torres-Carrasquillo, N. Dehak, E. Godoy, D. Reynolds, F. Richardson, S. Shum, E. Singer, and D. Sturim, "The MITLL NIST LRE 2015 Language Recognition System," in Odyssey (to appear), 2016.

[24] A. Coates and A. Y. Ng, "Learning Feature Representations with K-Means," in Neural Networks: Tricks of Trade, 2012.

[25] INRIA, "SPArse Modeling software (SPAMS v2.5)," http://spams-devel.gforge.inria.fr/.

[26] R. Rubinstein, M. Zibulevsky, and M. Elad, "Efficient Implementation of the K-SVD Algorithm using Batch Orthogonal Matching Pursuit," http://www.cs.technion.ac.il/rronrubin/software.html, Tech. Rep., 2008.

[27] C.-C. Chang and C.-J. Lin, "LIBSVM: Library for Support Vector Machines," ACM Trans. on Intelligent Systems and Technology, 2011. 